\title{
Dispositivos móviles: el nuevo reto para la industria de la prensa y del libro en España
}

\section{Carmen Costa-Sánchez ${ }^{1}$ Ana Isabel Rodríguez-Vázquez² Xosé López-García ${ }^{3}$}

Recibido: 2015-06-16

Enviado a pares: 2015-06-18
Aprobado por pares: 2015-07-29

Aceptado: 2015-08-05

DOI: 10.5294/pacla.2016.19.2.8

Para citar este artículo / to reference this article / para citar este artigo Costa-Sánchez, C., Rodríguez-Vázquez, A. I. \& López-García X. (Junio de 2016). Dispositivos móviles: el nuevo reto para la industria de la prensa y del libro en España. Palabra Clave, 19(2), 526-555. D0I: 10.5294/pacla.2016.19.2.8

\section{Resumen}

El presente artículo analiza los cambios que introducen los nuevos dispositivos móviles en las industrias culturales más tradicionales europeas: la industria de la prensa y la editorial. Tras evaluar la situación actual de ambos sectores en España, reflexiona sobre el cambio real que se está produciendo en el ecosistema mediático, debido a la irrupción de la denominada cuarta pantalla. Los datos auguran cambios más acelerados para la industria de la prensa que para la del libro.

\section{Palabras clave}

Prensa; industria editorial; periodismo; dispositivos móviles (Fuente: Tesauro de la Unesco).

\footnotetext{
Universidad de Coruña. España. carmen.costa@udc.es

Universidad Santiago de Compostela. España. anaisabel.rodriguez.vazquez@usc.es

Universidad Santiago de Compostela. España. xose.lopez.garcia@usc.es
} 


\section{Mobile Devices: The New Challenge for the Newspaper and Book Industry in Spain}

\section{Abstract}

This article analyzes the changes introduced by new mobile devices in more traditional European cultural industries: the newspaper and the publishing industry. After evaluating the current situation in both sectors in Spain, this reflects on the real change that is occurring in the media ecosystem, due to the emergence of the so-called fourth screen. The data predicts more accelerated changes for the press industry than for the book industry.

\section{Keywords}

Press; publishing; journalism; mobile devices (Source: Unesco Thesaurus). 


\section{Dispositivos móveis: o novo desafio para a indústria da imprensa e do livro na Espanha}

\section{Resumo}

Este artigo analisa as mudanças que os novos dispositivos móveis introduzem nas indústrias culturais mais tradicionais europeias: a indústria da imprensa e a editorial. Após avaliar a situação atual de ambos os setores na Espanha, reflete-se sobre a mudança real que está sendo produzida no ecossistema midiático devido à irrupção da denominada "quarta tela". Os dados preveem mudanças mais aceleradas para a indústria da imprensa do que para a do livro.

\section{Palavras-chave}

Imprensa; indústria editorial; jornalismo; dispositivos móveis (Fonte: Tesauro da Unesco). 


\section{Introducción: justificación del tema de estudio}

Los medios impresos (libros y diarios) se hallan inmersos en un proceso de reconversión. Hay tres factores que han provocado esta reconfiguración en marcha de la mano de la comunicación digital interactiva. La digitalización y las nuevas herramientas tecnológicas, la crisis económica que ha comenzado en 2008 en nuestro entorno y los cambios en los usos y consumos de los productos comunicativos (Meyer, 2006; Scolari, 2008; Curran, 2010; Picard, 2010; Salaverría, 2010).

A medida que pasan los años, los cambios en el ecosistema comunicativo resultan más relevantes. La convivencia papel/digital continúa bajo el predominio del modelo de negocio en papel, pero la tendencia se dirige hacia el consumo en diferentes pantallas.

Las bases industriales de los medios impresos sufren constantes sacudidas (Casero-Ripollés, 2010) que alimentan amenazas a su modelo de negocio. Diferentes informes de la evolución de los medios impresos tanto de los diarios como del libro apuntan al escenario convulso en el que se mueven y los desafíos a los que se enfrentan (WAN-IFRA, 2015; Observatorio del Libro y la Lectura, 2015; Corredor, 2013). La supervivencia de muchas empresas del sector está en juego (Díaz-Nosty, 2011), lo cual promueve la búsqueda de nuevos modelos de negocio y nuevos productos.

Es en esa búsqueda de alternativas para el futuro donde la industria se ha fijado en la irrupción de la comunicación móvil que ofrece un nuevo medio dotado de un ecosistema propio (Aguado, Feijóo y Martínez, 2013) y un renovado escenario en red (Castells et al., 2007), en el que los usuarios han acogido con interés informativo y de entretenimiento la denominada "cuarta pantalla" que, a causa de la disponibilidad y de la ubicuidad que brindan al usuario, la convierten en un elemento clave en el proceso de convergencia e integración de la comunicación digital (Aguado y Martínez, 2010).

El presente artículo reflexiona sobre la situación de la prensa y de la industria editorial en España para analizar qué ha cambiado o está 
cambiando gracias a la llegada de dispositivos móviles específicos, es decir, teléfonos inteligentes y tabletas en la prensa y e-readers para la industria editorial.

Para ello, en primer lugar, se estudiará su situación actual (la de ambas industrias) según los datos de las fuentes de referencia, y en un segundo momento se determinarán las potencialidades y limitaciones que ofrecen los nuevos soportes.

\section{Metodología}

En este estudio, se utiliza la revisión documental como metodología prioritaria, ya que es la que permite la reflexión sobre la evolución y la situación actual de la industria de la prensa y de la edición de libros, tomando como punto de partida los datos publicados por las fuentes primarias de referencia en España.

Como apuntan Rodríguez y Valldeoriola (2013, p. 25), "ante la gran cantidad de información disponible actualmente, cualquier investigador que se precie debe conocer las principales fuentes documentales, así como dominar los mecanismos de acceso, búsqueda, recopilación y organización de dicha documentación”.

La revisión documental es la técnica cualitativa empleada mayoritariamente en el análisis de las industrias culturales:

A pesar del rechazo que pueda producir en algunos círculos y autores, difícilmente podremos intervenir sobre la base de estas ideas si no conseguimos medir y visualizar el fenómeno, aunque sea de forma imperfecta. Los enfoques pueden ser cualitativos, cuantitativos 0 mixtos, con fuentes de datos primarias o secundarias, perspectivas de oferta 0 demanda, y sectoriales o espaciales (Boix y Lazzeretti, 2012, p. 186).

Por tanto, además de la literatura científica existente, se han analizado los principales informes sectoriales tanto de fuentes públicas como privadas, que pasamos a sintetizar para cada una de las industrias analizadas. 
En la industria de la prensa, se han revisado los libros blancos de la prensa diaria desde un año antes de la crisis, es decir: 2007, 2008, 2009, 2010, 2011, 2012, 2013 y 2014, que publica la Asociación de Editores de Diarios Españoles (AEDE). También se han tomado en cuenta diversos informes de la WAN (Asociación Mundial de Periódicos) y del Pew Research Center para una contextualización de tendencias más general. Asimismo, se han consultado los datos de OJD (Oficina de Justificación de la Difusión) y de EGM (Estudio General de Medios) como fuentes de referencia para el análisis de los datos de tirada, difusión y lectores en España.

En lo que respecta a la industria del libro, se han revisado los informes El sector del libro en España 2013-2015 y la Panorámica de la edición española de libros 2013, que elabora el Ministerio de Educación, Cultura y Deporte a través de su Observatorio del Libro y de la Lectura. También los informes de la Federación de Gremios de Editores Españoles (FGEE) sobre el Comercio interior del libro en España (2013), Comercio exterior del libro (2013) y Hábitos de lectura y compra de libros 2012 (el último publicado).

La primera hipótesis de la que se parte en el presente trabajo es que la industria de la prensa, a pesar de que ha incorporado los nuevos canales móviles como soportes informativos, todavía no elabora una producción periodística adaptada a las singularidades tecnológicas y de contenido de los nuevos dispositivos.

Una segunda hipótesis de partida recoge que, en la industria del libro, falta un proceso de expansión social de la tecnología específica en España, así como una mayor apuesta desde la industria editorial y desde las políticas culturales, lo cual está ralentizando el grado de éxito de la nueva plataforma.

La tercera de las hipótesis de partida sostiene que ambas industrias afrontan este mismo reto a ritmos distintos, más lento en la industria librera. 


\section{Situación actual de la industria del libro y de la prensa en España}

Las industrias culturales españolas padecen los efectos de la crisis económica que afecta a España (y a Europa), que es estructural y multidimensional (Castells, Caraça y Cardoso, 2013) y que tiene como principal consecuencia la debilitación, con destrucción parcial, del tejido productivo.

La caída del consumo interno, la drástica reducción de las ayudas púbicas y las dificultades para la financiación, junto con otros efectos de la reconfiguración que implica la globalización en un escenario neoliberal, han colocado a la mayor parte de las industrias culturales ante un callejón de difícil salida (Celaya, Rausell y Villarroya, 2013). El panorama es en especial crítico para la industria del libro y de los medios impresos.

\section{La industria de la prensa}

El contexto actual de la prensa en España se encuentra marcado por tres procesos de cambio y sus correspondientes derivaciones (AEDE, 2014):

1. Una disminución del número de cabeceras en papel, que llegó a lo largo de la década a su momento álgido en 2007 con 140 cabeceras diarias impresas, pero en la actualidad se contabilizan 113. El 2008, considerado de incidencia de la crisis económica, inaugura el cierre en cascada de diarios en papel.

Las cabeceras digitales, por su parte, experimentan un crecimiento en número medio hasta 2010 (cuando se llega a las 134 cabeceras digitales de media; desde entonces, desciende en menor medida hasta las 125).

2. Una fuerte bajada del volumen de su difusión y de la inversión publicitaria, tema que se abordará a fondo más adelante.

3. Un cambio en los hábitos de lectura del lector tradicional de prensa en papel, que migra cada vez más hacia los soportes en línea y los distribuidores o agregadores de noticias. 
La crisis de lectores de prensa en España comenzó en 2005. Después de dos años seguidos en los que el número de lectores de diarios se había incrementado de forma muy notable, 2005 supuso una primera bajada, pues de los 13.9 millones de lectores en 2004 se pasa en 2005 a una cifra de 13.5 millones (AEDE, 2007). Los síntomas de un cambio de tendencia comenzaban a advertirse.

La difusión de la prensa diaria, según la patronal del sector, era en España en 2001 de 4274323 ejemplares, mientras que en 2013 apenas alcanza los 3008257 de ejemplares, lo cual supone una pérdida acumulada de 1.2 millones de periódicos en doce años.

Los datos de la OJD apuntan a que en 2004 se alcanzó el máximo índice de difusión de la prensa en España, con más de 4 millones difundidos cada día (exactamente, 4117353 ejemplares por día). Ocho años después, en 2012, en plena crisis económica y crisis de lectura, esa cifra estaba en poco más de la mitad: 2899556 ejemplares (tabla 1).

\section{Tabla 1. Evolución de la difusión de los 10 diarios españoles de información general más vendidos (2008-2014)}

\begin{tabular}{|l|c|c|c|}
\hline \multicolumn{1}{|c|}{ Diarios } & 2008 & 2014 & $\begin{array}{c}\text { Diferencia } \\
(-)\end{array}$ \\
\hline El País & 431033 & 259775 & 171258 \\
\hline El Mundo & 323587 & 149684 & 173903 \\
\hline La Vanguardia & 201859 & 140176 & 61683 \\
\hline ABC & 251642 & 128660 & 122982 \\
\hline La Razón & 154410 & 81449 & 72961 \\
\hline El Periódico de Catalunya & 152025 & 92163 & 32862 \\
\hline La Voz de Galicia & 102858 & 78414 & 24444 \\
\hline El Correo & 115365 & 77237 & 38128 \\
\hline El Diario Vasco & 80095 & 55923 & 24172 \\
\hline La Nueva España & 58917 & 46183 & 12734 \\
\hline
\end{tabular}

Fuente: elaboración propia.

Mientras en 2002, 97 de cada 1000 españoles leía algún periódico, en 2012 esa cifra se situaba en 64 ciudadanos de cada 1000. "Hay que dejar 
constancia que situarse por debajo del nivel 100 es la cifra a partir de la cual la Unesco considera propia de países subdesarrollados" (Marqués Pascual, 2012, p. 34). La caída ha colocado a España en el penúltimo lugar del escalafón en Europa en este ámbito, ya que solo Portugal, con 53 de cada 1000, ofrece peores índices (AEDE, 2014).

En datos del EGM (http://www.aimc.es/-Datos-EGM-ResumenGeneral-.html), el porcentaje de penetración de los diarios sobre la población española presenta las cifras más bajas de la última década. Los datos más actualizados (según la última oleada, abril de 2014 a marzo de 2015 ) muestran un índice de penetración de $29.1 \%$, mientras que el punto álgido se alcanzaba en 2008 con $41.8 \%$. De nuevo, 2008 marca el inicio del declive.

$\mathrm{Al}$ contrario, la penetración de la prensa digital muestra una tendencia de crecimiento que no ha dejado de aumentar desde el inicio de la década. Los lectores que combinan papel y digital evolucionan en positivo, aunque más paulatinamente. El perfil sociodemográfico del lector digital es el de un lector varón más joven que el de la prensa en papel (menor de 44 años) y su edad aproximada se sitúa en torno a los 39 años (AEDE, 2014) (figura 1).

\section{Figura 1. Penetración de lectores en prensa: papel, digital y ambos}

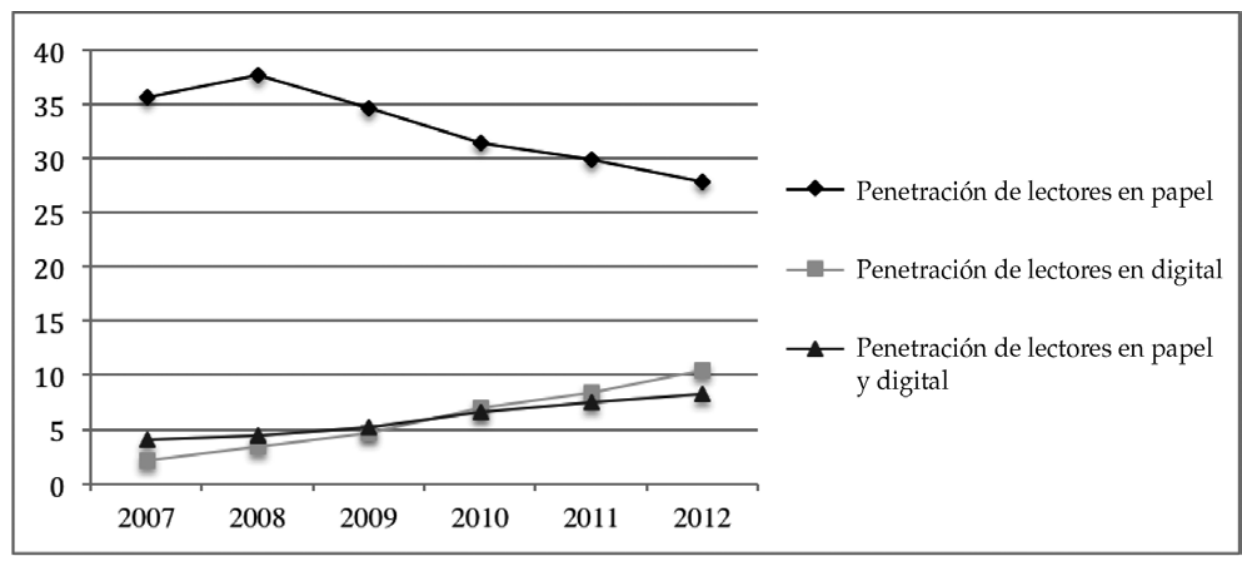

Fuente: elaboración propia a partir de AEDE (2014). 
Además, en coherencia con la bajada de la difusión, los ingresos de los diarios de pago son cada vez más reducidos. La venta en quiosco es cada vez menor, mientras que la lectura por ejemplar crece, lo cual indica que las personas que tienen el hábito de leer un periódico en papel lo mantienen, pero encuentran otra manera de hacerlo sin tener que pagar (Marqués, 2012, p. 314).

A esta pérdida de ingresos hay que añadir el descenso en los ingresos publicitarios, que ha afectado el conjunto de los diarios y ha sido superior a $25 \% .^{4}$ La inversión publicitaria en prensa alcanzaba en 2007 los 1848 millones de euros, punto de máxima inversión de la década, que a partir de ahí decrecía hasta llegar a los 731 millones de euros en 2012. La inflexión tiene lugar a partir de 2008 (tabla 2).

\section{Tabla 2. Evolución de la inversión publicitaria en prensa en España en millones de euros}

\begin{tabular}{|c|c|c|c|c|c|c|}
\hline Año & 2007 & 2008 & 2009 & 2010 & 2011 & 2012 \\
\hline $\begin{array}{l}\text { Inversión publicitaria en prensa } \\
\text { en millones de euros }\end{array}$ & 1848.7 & 1490.6 & 1117.9 & 1057.4 & 930 & 731.1 \\
\hline
\end{tabular}

Fuente: elaboración propia a partir de AEDE (2014).

Las consecuencias de todo ello van más allá de las cifras, pues ha afectado a las empresas informativas, a las condiciones laborales de los periodistas y del producto que elaboran. Según Campos (2010), una de estas consecuencias ha sido la descapitalización de las empresas periodísticas y los cambios en las estructuras de propiedad de los principales grupos, que se han visto obligados a la venta de activos y a permitir la entrada de nuevos socios procedentes de los sectores financieros más especulativos (fondos de inversión).

La situación se ha resuelto frecuentemente con el despido masivo de periodistas, el cierre de corresponsalías y servicios externos, la reducción en el número de páginas y el recorte en la amplitud y variedad de las agendas. Esto es, con pérdida de calidad, que incluso se

4 Los datos proceden de los informes ofrecidos por el Libro Blanco de la Prensa Diaria en España de 2010, 2011, 2012 y 2013. 
ha rubricado en algunos casos con la elevación del precio del ejemplar impreso (Díaz-Nosty, 2011, p. 56).

Al mismo tiempo, en un futuro que ya es presente, los soportes móviles se configuran como una nueva oportunidad para recuperar lectores e interés por la información, con una oferta diferenciada en la adaptación a las características de los nuevos medios.

\section{La industria del libro en España}

Los índices de lectura en España, aunque mostraron alguna debilidad en la segunda mitad de la primera década de 2000, puesto que los datos de 2009 indicaban que el índice de lectura se situaba en $54.6 \%$, lo cual implicaba un descenso de 2.2 puntos en relación con las cifras de 2007 (Federación de Gremios de Editores de España [FGEE], 2008), se incrementaron en la última década hasta alcanzar $63 \%,{ }^{5}$ lo cual se ha explicado por el tiempo libre que deja la crisis y el paro (Federación de Gremios de Editores de España, 2008).

En cambio, el comercio interior del libro en papel ha mantenido una tendencia a la baja, de forma que los 2182 millones de euros de ventas en comercio interior en 2013 retrotrae al sector a mediados de la década de 1990 (Barandiarán, Gil y Ortuño, 2014, p. 53).

En la comparativa por soportes, la edición de libros en soporte papel se ha reducido, en 2013, $17.7 \%$, mientras que la edición en otros soportes ha descendido $5.9 \%$ (Observatorio de la Lectura y el Libro, 2014). En 2014 creció por primera vez en los últimos cinco años 3.7 \% la edición en papel, aunque, con respecto a 2009, la edición de libros en este soporte ha registrado un descenso de $29.5 \%$, bajada muy superior a la experimentada por el total de la edición, que ha sido de $17.6 \%$ (Observatorio de la Lectura y el Libro, 2015) (figura 2).

5 El porcentaje de $63 \%$ de la población que lee, ofrecido en el Barómetro de Hábitos de Lectura y Compra del Libro, ha sido ratificado por estudios posteriores, en especial por el estudio recogido en el Barómetro del Centro de Investigaciones Sociológicas (CIS) de diciembre de 2014, donde figura un apartado dedicado a los hábitos de lectura de los españoles (http://www.cis.es/cis/opencms/ES/NoticiasNovedades/InfoCIS/2015/Documentacion_3047.html). 


\section{Figura 2. Producción del libro por soportes}

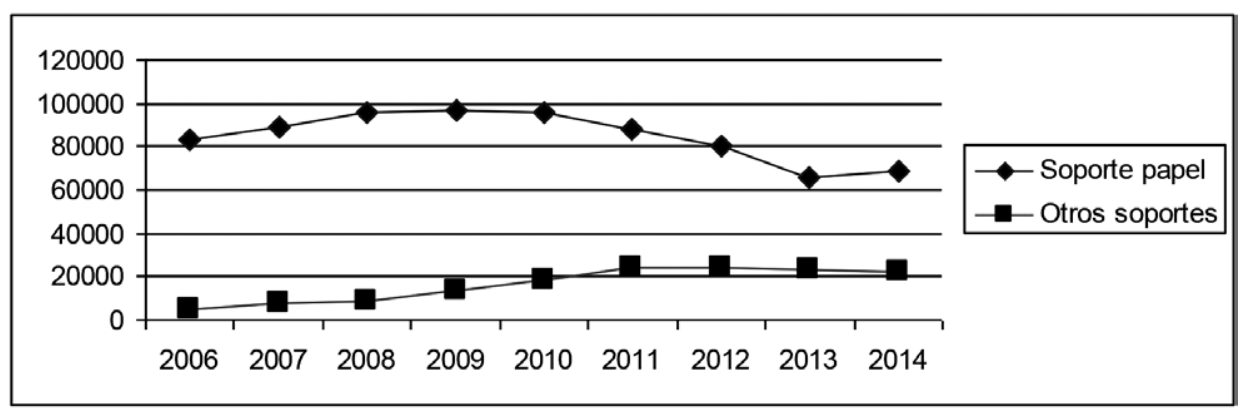

Fuente: elaboración propia a partir de Observatorio de la Lectura y el Libro (2015).

La facturación del sector editorial en el mercado interior también ha descendido en $201311.7 \%$, lo cual ha supuesto la acumulación de cinco años consecutivos de caídas. Los informes del Comercio interior del libro en España señalan que entre 2009 y 2013 la cifra de facturación en el mercado interior ha disminuido $29.8 \%$ y el número de ejemplares $34.9 \%$ (Observatorio de la Lectura y el Libro, 2015) (figura 3).

\section{Figura 3. Facturación del libro en España}

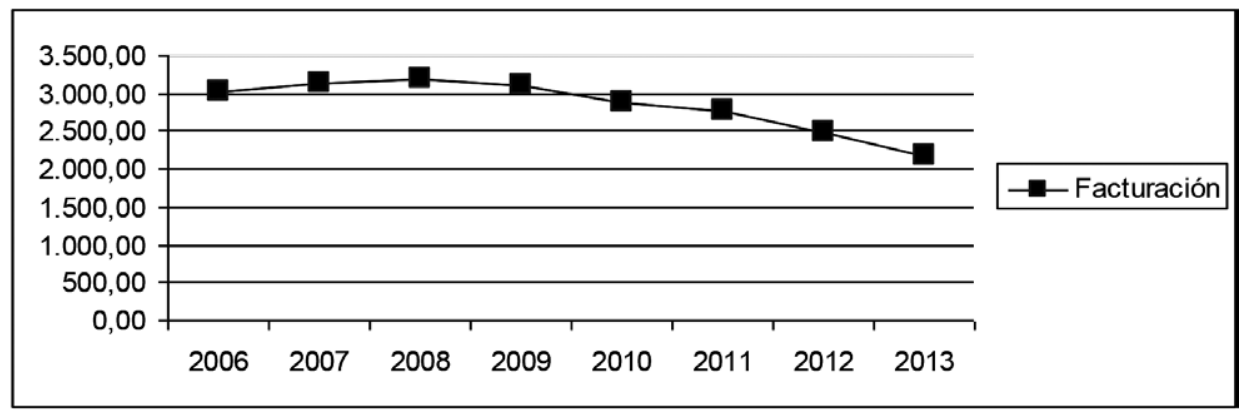

Fuente: elaboración propia a partir de Observatorio de la Lectura y el Libro (2015).

Donde mantiene buenas posiciones y una tendencia positiva el libro español es en el comercio exterior. En España, que junto con el Reino Unido y los Estados Unidos lidera la exportación de libros en el mundo, el saldo neto del sector en 2013 ha superado los 322 millones de euros, lo que representa $8 \%$ más que 2012 . 
El valor de las exportaciones creció $7.3 \%$, e Iberoamérica, con México a la cabeza, es el lugar preferente de destino de los libros españoles (Observatorio de la Lectura y el Libro, 2015).

En este escenario de pérdida de protagonismo del papel, la revolución digital, que no conoce fronteras, obliga al sector del libro a reciclarse y afrontar los desafíos del futuro, para lo que se precisa una política activa porque el mercado por sí solo no garantiza la difusión de la diversidad y la pluralidad cultural (Gómez-Escalonilla, 2005).

Esa política para el sector, que cuenta con tradición en España, busca combinar el apoyo a la edición en papel y a la edición digital, que se considera una opción para la diversificación. A pesar de que el sector considera con muchas cautelas el libro digital, existe cierto optimismo entre los editores (Gómez-Escalonilla, 2012) porque entienden que el futuro del libro es la convivencia del papel y el digital. El paso del tiempo parece darles la razón, aunque lo digital sigue creciendo y las ventas en papel descendiendo.

\section{Prensa y dispositivos móviles}

En 2014 España consolida su liderazgo dentro de los países más relevantes de la Unión con $81 \%$ de teléfonos sobre el total de móviles, 10 puntos por encima de la media y, además, dos de cada tres españoles utilizan más de un dispositivo para acceder a internet, por encima del Reino Unido y de los Estados Unidos, de forma que 23 millones de usuarios activos de aplicaciones realizan 3.8 millones de descargas diarias de aplicaciones (VV.AA., 2015).

Además, por primera vez, el tiempo de acceso a medios digitales dedicado desde dispositivos en movilidad supera al empleado desde el PC (53 frente a 47 \%), según el informe La sociedad de la información en España 2014 (Fundación Telefónica, 2014).

Los dispositivos móviles (tabletas y teléfonos) se están consolidando como herramienta para informarse. 
En datos del estudio How mobile devices are changing community information environments, del Pew Research Center, se recoge que $47 \%$ de los estadounidenses adultos usan sus teléfonos y tabletas para obtener noticias locales e información.

En datos del VI Estudio Anual IAB Spain Mobile Marketing, de IAB Spain Research y The Cocktail Analysis, 9 de cada 10 internautas tiene un teléfono inteligente, creciendo significativamente el segmento de 36 a 55 años. El móvil gana a la tableta como segunda pantalla preferida por los españoles. Leer la prensa es la cuarta actividad realizada a diario en internet móvil después de chatear, usar el correo electrónico y acceder a redes sociales.

Aunque los primeros estudios sobre el fenómeno de la comunicación móvil se centraron en trabajos de índole cultural y sociológica, a partir de 2004 se empiezan a considerar sus posibilidades como medio de información, y es cuando los investigadores incorporan este ámbito de estudio a sus trabajos (Silva Rodríguez, 2013).

Todo cambio tecnológico en los medios de comunicación implica una adaptación en las estructuras, los usos, los lenguajes y las posibilidades que de ello derivan. Así, al igual que el ciberperiodismo pasó por distintas etapas desde su arranque a mediados de la década de 1990 hasta hoy (y continúa su evolución), también el periodismo móvil ha pasado por diferentes estadios hasta su configuración actual, y es el momento de analizar en qué situación se encuentra y cuáles son las tendencias de futuro que afronta.

Según Westlund (2013, p. 23), investigador de referencia en este ámbito, "continuarán ideándose nuevos servicios en los medios móviles y mientras la gente los usa de formas que ahora no podemos imaginar, se convertirán en algo asentado en nuestro día a día”.

Los dispositivos móviles suponen un reto importante para las empresas periodísticas a la hora de producir contenidos capaces de ser transmitidos en un nuevo contexto en el que la información se consume en movilidad: desde cualquier lugar y en cualquier/todo momento. 
La "cuarta pantalla" presenta una serie de características que le permiten adaptarse al nuevo entorno y cumplir con las necesidades que demanda la sociedad. Las características principales del nuevo medio (Scolari et al., 2009) engloban: integración multimedia, personalización, geolocalización como una subcategoría de personalización y participación de los usuarios (o socialización). Sánchez y Aguado (2010) opinan que la llegada del móvil provoca una transformación en el concepto original de periodismo desde el punto de vista de la estructuración, con la aparición de nuevas modalidades expresivas. Vacas (2007) o Ahonen (2008) también lo consideran un nuevo medio.

El potencial informativo del nuevo medio ya ha sido pronosticado y comenzado a analizarse. Investigaciones previas han realizado aproximaciones prácticas al estado de la cuestión en análisis puntuales o muestrales de marcas periodísticas y soportes en España y Portugal (Costa-Sánchez, 2014; Silva Rodríguez, 2013; Costa-Sánchez, 2013; Sanjuán, Nozal y González, 2013; Canavilhas, 2009).

De los análisis efectuados por Costa-Sánchez (2014) o Silva Rodríguez (2013), se diagnostica que es en los elementos de personalización, geolocalización, integración multimedia y participación donde se hallan las principales carencias de las aplicaciones desarrolladas hasta la fecha por los diarios españoles y que es en estos cuatro ámbitos donde debe trabajarse en la creación de un nuevo modelo informativo para los móviles inteligentes. Guallar (2013) explica que en líneas generales las versiones para tabletas de los medios todavía no destacan ni por ofrecer contenidos propios o diferenciados ni por diseños en especial novedosos que las diferencien claramente de las ediciones en papel y web, siendo bien patente la dependencia de la edición papel. Meso et al. (2014) explican que, respecto del producto, las aplicaciones móviles encuentran su principal competencia en los propios sitios web clásicos de los medios, a los que se puede acceder igualmente a través de los navegadores incorporados en los dispositivos móviles.

El proceso está en marcha y resulta cambiante. Según Silva Rodríguez (2013), la importancia de las ediciones móviles se manifiesta, por ejemplo, en la visibilidad que dichas versiones tienen en la web de cada medio, de 
forma que más de $70 \%$ de las marcas de prensa analizadas incluyen dicha información en sus respectivas web (tabla 3).

\section{Tabla 3. Síntesis de estudios cuantitativos sobre prensa móvil en España}

\begin{tabular}{|c|c|c|c|}
\hline Autor/es & Objeto de estudio & $\begin{array}{c}\text { Muestra de estudio } \\
\text { analizada* }\end{array}$ & $\begin{array}{c}\text { Aplicaciones móviles } \\
\text { sobre la muestra }\end{array}$ \\
\hline Meso et al. (2014) & $\begin{array}{c}\text { Interactividad en web y aplicaciones de } \\
\text { prensa, radio y TV }\end{array}$ & 16 \\
\hline Costa-Sánchez (2014) & Aplicaciones para teléfonos inteligentes & 20 & 17 \\
\hline Silva Rodríguez (2013) & Web móvil y aplicaciones (sistema iOS) & 14 & 10 \\
\hline (Españoles y otros) & 14 & 10 & 29 \\
\hline Costa-Sánchez (2012) & Aplicaciones de medios nativos digitales & 29 & \\
\hline Canavilhas (2009) & Aplicaciones iPhone & & \\
\hline
\end{tabular}

*Si la muestra del estudio comprende diferentes tipos de medios, lo que se indica es la cifra de cabeceras de prensa que la integran.

Fuente: elaboración propia.

Según detecta Canavilhas (2012):

En un momento en el que las empresas de comunicación social atraviesan una de las peores crisis de la historia, el aumento de las tasas de penetración de smartphones y tablets puede constituir una fuente alternativa de ingresos. La posibilidad de ofrecer información hipermultimediática, contextualizada y personalizada responde a los deseos manifestados por los lectores cuando son preguntados sobre qué les llevaría a pagar por la información online (p. 16).

En coherencia, el proceso de creación de un nuevo producto periodístico para el nuevo medio ya está en marcha con la necesaria reconversión de las redacciones y de las dinámicas informativas de los periodistas. La cuestión ahora se centra en cómo definir un contenido diferenciado y rentabilizar el/los producto/s generado/s para las nuevas pantallas. Mientras que, por el momento, las marcas de prensa española apuestan mayoritariamente por la gratuidad e inserciones publicitarias (Costa-Sánchez, 2014) en el ámbito internacional, se está buscando el éxito de un modelo mixto (freemium) por medio de la suscripción como fórmula preponderante (Díaz-Noci, 2010; AEDE, 2014). En España los casos paradigmáticos de 
este tipo son los de los quioscos digitales, Orbyt (Unidad Editorial) y Kiosko y Más (Vocento/Prisa), que ofrecen .pdf enriquecidos de los medios impresos accesibles desde ordenadores, tabletas y teléfonos inteligentes.

Las empresas periodísticas deben encontrar soluciones únicas que hagan frente a la bajada en la difusión, la lectura de diarios y el descenso en los ingresos publicitarios (Picard, 2010). Aun sin un modelo claro de contenido, expresivo y de negocio, que optimice las potencialidades del nuevo medio, los dispositivos móviles se han convertido en el foco de atención de las estrategias actuales.

\section{Libros electrónicos y dispositivos móviles}

El gran salto en la producción de libros digitales en España se produce en el bienio 2009-2010, cuando se pasa de una producción de 5077 libros digitales a los 12948 del año siguiente. El interés de la industria española por complementar el papel con el negocio digital quedaba patente.

Sin embargo, desde 2012 se detecta un leve descenso que se mantiene en los últimos datos. Es la primera vez que se invierte la tendencia hacia el aumento de la edición digital desde 2008. En concreto en 2014 se publicaron 20263 libros digitales en España, 1.9 \% menos que en el año anterior.

Sobre el total de libros producidos en España en 2014, los libros digitales suponen $22.3 \%$, lo cual muestra que lo principal del negocio de la industria librera española se produce del papel.

Sobre el total de empresas con actividad editorial en España en 2014, menos de la cuarta parte, $21.9 \%$ publicó libros en digital (Observatorio de la Lectura y el Libro, 2015). La tendencia es cuatro puntos porcentuales menos que el año anterior (tabla 4).

La facturación, al contrario, va en aumento, en un contexto de caída de facturación general. Según el informe del Comercio interior del libro en España 2013, la facturación del libro digital alcanzó el pasado ejercicio los 80.3 millones de euros, mostrando en el último año un incremento de $8.1 \%$. El crecimiento en los últimos cuatro años alcanza $13.9 \%$. 


\section{Tabla 4. Número de libros digitales producidos en España (2008-2014)}

\begin{tabular}{|c|c|c|c|c|c|c|}
\hline 2008 & 2009 & 2010 & 2011 & 2012 & 2013 & 2014 \\
\hline 2519 & 5077 & 12948 & 18339 & 20708 & 20655 & 20263 \\
\hline
\end{tabular}

Fuente: elaboración propia a partir de Observatorio de la Lectura y el Libro (2014).

En cuanto a los soportes, tal y como se recoge en El sector del libro en España 2013-2015, la modalidad de consumo de libros en streaming, por tanto, sin la necesidad de descargarse el contenido en un dispositivo, va incrementándose llamativamente, de forma que de $4.4 \%$ que supuso en 2012 asciende a $48.6 \%$ en 2013.

Al contrario, el denominado e-reader, es decir, el dispositivo electrónico diseñado principalmente para la lectura digital de libros y que utiliza tecnología e-ink para presentar el contenido a los lectores (Burk, 2001, p. 326), decrece en importancia desde la apuesta editorial, y representa $21.6 \%$ del total de la oferta. La tableta crece ligeramente $(0.9 \%)$ y supone $6.1 \%$ del total (tabla 5).

\section{Tabla 5. Dispositivos de lectura para los que se comercializan las obras digitales}

\begin{tabular}{|c|c|c|c|}
\hline Dispositivo & $2012(\%)$ & $2013(\%)$ & Diferencia (\%) \\
\hline Lectores específicos & 38.1 & 21.6 & $(-) 16.5$ \\
\hline Tabletas & 5.2 & 6.1 & $(+) 0.9$ \\
\hline Móviles/PDA & 1.8 & 1 & $(-) 0.8$ \\
\hline Ordenadores & 49.2 & 21.4 & $(-) 27.8$ \\
\hline Streaming & 4.4 & 48.6 & $(+) 44.2$ \\
\hline Otros & 1.3 & 1.3 & $(=) 0$ \\
\hline
\end{tabular}

Fuente: elaboración propia a partir de Federación de Gremio de Editores de España (2013).

Por formatos, el formato ePub continúa ganando cuota de presencia (4.1\% más que el año anterior), en paralelo al descenso continuado del formato .pdf o MOBI (Kindle). En referencia a las temáticas de los libros digitales, texto no universitario y ciencias sociales y humanas, se consolidan como las materias que generan la mayor parte de la facturación del libro digital (58 \% del total de la facturación entre ambas materias) (figura 4). 


\section{Figura 4. Libros digitales 2014 por géneros}

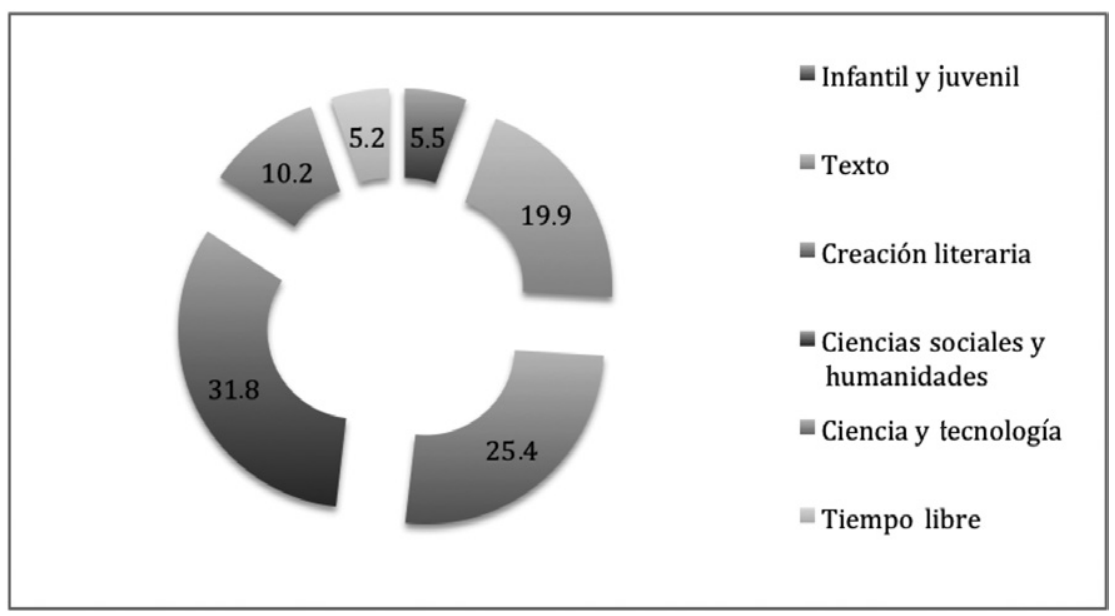

Fuente: elaboración propia a partir Observatorio de la Lectura y el Libro (2015).

Según el informe de Hábitos de lectura y compra de libros que realiza la FGEE, el índice de lectores en digital en España alcanza 58 \%, seis puntos porcentuales más que el año anterior. Además, los lectores en digital han aumentado en todos los soportes. En el ordenador, 9.3 puntos; en el móvil y agenda electrónica, 6 puntos. El aumento más significativo se produce en la lectura en el e-reader, que es cinco veces mayor que la registrada en 2010.

Según los datos sobre equipamiento tecnológico que proporcionan los estudios de IAB sobre mobile marketing, tanto la tableta como los e-readers no han dejado de incrementar su índice de penetración en España. En 2012 se situaba para ambos dispositivos en $23 \%$. En 2013 la tableta aumentaba a $43 \%$ y a $57 \%$ en 2014, lo cual significa que prácticamente seis de cada diez españoles disponen de una. Los datos para los e-readers dejan de ser segmentados en estudios posteriores. Pero el Instituto Nacional de Estadística (INE), en su Encuesta sobre equipamiento y uso de tecnologías de la información y comunicación en los hogares 2014, indica que $20 \%$ de los hogares españoles tiene un lector de $e$-books, en especial en aquellos con unos ingresos mensuales netos superiores a los 2500 euros, cuando la cifra alcanza $42 \%$. 
Acerca del estudio del e-reader como nuevo medio, es decir, como nuevo soporte lector, debe tenerse en cuenta su reciente irrupción en el mercado, pues es en 2007 cuando puede hablarse del despegue de los dispositivos de lectura electrónicos de nueva generación con pantallas de tinta digital, siendo las plataformas de Amazon (Kindle) y el modelo de Sony las marcas que lideran el proceso (Cordón, Arévalo y Martín, 2010).

En un análisis de los dispositivos, Siegenthaler, Wurtz y Groner (2010) han detectado fallos en la usabilidad del dispositivo, por lo que recomendaban algunas mejoras para los próximos modelos, entre las que figuraba la planificación de un diseño más intuitivo, ya que los usuarios esperan más de ese dispositivo al margen de que sirva de soporte textual.

De especial interés son los resultados del trabajo de Jung et al. (2012), quienes, tras el estudio de recepción correspondiente, concluyen que los usuarios que más gratificaciones obtienen de los medios impresos son también, paradójicamente, los más propensos al uso dele-reader. De esta forma, demostraban el papel suplementario pero no sustitutorio del nuevo medio, puesto que no se da un efecto de desplazamiento del papel por la tinta electrónica, sino de coherencia en consumo de medios.

En el estudio de recepción de Maynard (2010), los adultos mostraban su preferencia por los libros en papel, mientras que los niños eran más ambivalentes, dividiéndose entre ambas preferencias.

Atendiendo al diagnóstico del futuro del libro digital, según GómezEscalonilla (2012), la situación del libro digital en España es complicada porque no hay contenidos atractivos. Otro de los obstáculos que afronta el libro digital en España es el precio, nada favorecido por el IVA. A pesar de que la Ley 10/2007 de la lectura, del libro y de las bibliotecas, que revoca la Ley de 1975, reconoce al libro digital, al considerarse un servicio electrónico, le corresponde el tipo general y no el reducido (como ocurre con el libro en papel), lo cual grava la compra con $21 \%$ de IVA en lugar de $4 \%$. La ausencia de leyes de precio fijo en otros países no contribuye a la hora de plantearse las posibilidades del mercado español en la esfera internacional. 
Costa-Knufinke (2010) recomienda a las editoriales españolas sacar partido a las ventajas del $e$-book al permitirles abrir un nuevo canal de distribución inmediato, permanente y con gran potencial, la actualización constante del catálogo y el cross publishing, es decir, la edición de un mismo contenido en diferentes formatos según las necesidades del mercado. Asimismo, apuesta por la elaboración de productos complejos que usen todos los instrumentos multimedia disponibles para ayudar a evitar la piratería.

\section{Conclusiones}

Los dispositivos móviles están abriendo un nuevo panorama para dos de las industrias culturales más tradicionales en España y en Europa: la industria periodística y la editorial. Las novedades de los dispositivos específicos que los avances tecnológicos han generado permiten una nueva relación interfaz-usuario con el producto cultural, que parece animar el consumo. No obstante, conviene diferenciar la situación de la industria de la prensa, respecto de la del libro, ya que existen magnitudes que permiten hablar de horizontes distintos para cada una de ellas.

Así, la industria de la prensa española está realizando una apuesta clara por los nuevos dispositivos, aunque todavía se ensaye la manera de crear un producto que se diferencie de la web según las singularidades del nuevo medio. Personalización o geolocalización de contenidos, por ejemplo, son estrategias de interés que no se están empleando. La adaptación en las estrategias expresivas e informativas es otra de las cuestiones clave, a la que además todavía se le está prestando una atención incipiente desde la investigación (Costa-Sánchez, Rodríguez-Vázquez y López-García, 2015).

Lo que cabe concluir es que la evolución de lo en línea a lo en línea y móvil está en marcha. Los tres productos: prensa en papel, prensa digital y prensa móvil ya conviven en la sociedad española, aunque esta última no acabe de definirse en cuanto a modelo periodístico, de contenido y de negocio.

La industria de la prensa se ha visto muy afectada por la crisis económica de 2008 y por una crisis estructural en el planteamiento de un modelo de negocio que se encuentra en decadencia. Por ello, dado el éxito 
social de los nuevos dispositivos, ensaya la fórmula más adecuada para rentabilizar la plataforma no solo en términos económicos, sino de consumo informativo. Los lectores más jóvenes son lectores digitales y en su mayoría disponen de un dispositivo móvil en el bolsillo, así que la combinación, si se sabe aprovechar, parece un camino de futuro que podría conducir al éxito.

En el caso de la industria editorial, el hecho de que la facturación crezca en cuanto al libro digital, así como el creciente número de lectores, se constituye en el principal argumento para que la industria apueste estratégicamente por este ámbito.

Pero el proceso, en cuanto al libro digital se refiere, avanza con cautela. En número, los libros digitales y las empresas que los editan experimentan un leve retroceso, además de que el género predominante todavía es el libro de texto y no el de ficción (o creación), lo cual reflejaría una mayor madurez en el mercado. El papel reclama su dominio ante la tinta electrónica.

Algunos de los motivos que están detrás de esta cuestión es una adopción social menor de la nueva tecnología en comparación con el teléfono inteligente y también con respecto a la tableta si se quiere prestar atención al dispositivo de lectura específico (e-reader).

Por otra parte, la producción editorial en digital supone menos de una cuarta parte de la producción editorial de conjunto, lo cual da idea de que la convivencia papel/e-ink en desigualdad de condiciones se mantendrá a largo plazo. "Las editoriales españolas $[. .$.$] todavía no editan en digital"$ (Gómez-Escalonilla, 2012, p. 560). La investigación confirma, por tanto, su triple hipótesis de partida: la industria de la prensa, ante un panorama de gran adopción social de la nueva tecnología, ya es prensa móvil, aunque todavía no acomete una producción periodística adaptada a las singularidades y ventajas del nuevo medio.

La industria del libro, por su parte, todavía se encuentra en una etapa incipiente. La tecnología específica presenta unas tasas de penetración so- 
cial limitadas, y aunque lo digital sigue una tendencia global creciente en la producción y el negocio, la producción editorial continúa estando bajo el reinado del soporte papel.

Ambas industrias, por tanto, afrontan este reto a distintas velocidades.

De lo analizado, puede concluirse también que los nuevos medios no compiten por expulsar a los "viejos". Convivencia e interinfluencia serían las dos palabras que mejor pueden definir el fenómeno.

En la industria de la prensa, las aplicaciones para teléfonos inteligentes se ven favorecidas por la existencia de una marca periodística consolidada, que es la que ampara el conocimiento que tiene el usuario del nuevo producto. Por otra parte, los dispositivos móviles podrían ayudar a solventar el problema de indefinición del modelo de negocio y la predominante cultura de la gratuidad que existe en España asociada a internet. El nuevo medio representa una vía más proclive a desarrollar modelos freemium, como prueban los modelos de suscripción que ya existen en España o los que están desarrollando las cabeceras de referencia internacional, como The Guardian (Reino Unido) o The New York Times (Estados Unidos).

Refiriéndonos a la industria editorial, el libro digital es un complemento secundario del libro impreso y falta una expansión social mayor de la tecnología específica para la lectura de dichos nuevos formatos. Los medios no evolucionan por una selección natural, sino por una elección humana. El medio que mejor evoluciona es aquel que se ajusta más a las diversas necesidades del hombre (Islas-Carmona, 2008).

Por eso, comparativamente, teléfono móvil, teléfono inteligente y tableta sobresalen en los dispositivos móviles.

Pero el libro digital no aspira a sustituir al otro. "No se trata de postular la sustitución, sino de localizar el espacio que ha quedado hueco, seguir la distribución de vacíos y rupturas y vigilar las aperturas que esta desapa- 
rición deja entrever" (Cordón, Gómez y Alonso, 2011, p. 40). El gusto por la lectura, que cultiva el libro en papel, favorece el interés por el e-reader y el libro digital. Como en la prensa, el medio tradicional ampara al nuevo medio. Desde finales del siglo XX, los expertos entienden que hay campos en los que difícilmente el soporte digital sustituirá totalmente al papel (Codina, 1998).

La coordinación de potencialidades entre ambas plataformas es la clave. Sin querer augurar soluciones milagrosas para una situación complicada en ambas industrias culturales, la cuarta pantalla supone una actualización tecnológica con múltiples ventajas asociadas: nuevos lectores, nuevas vías de financiación, abaratamiento de costes de producto, nuevos servicios, bajo una fórmula de tiempos y espacios amoldable a las preferencias del usuario. Según Díaz-Nosty (2013, p. 85), "con los dispositivos móviles, el medio adquiere su continuidad distintiva; el medio se hace mensaje, se hace medio continuo".

Pero una perspectiva exclusivamente tecnologicista no dejaría ver el problema de estudio en toda su complejidad. El contenido es el reto que afrontan. Nuevos lenguajes narrativos, nuevos papeles para el prosumidor, nuevas formas de participación y de consumo social son tendencias que van asociadas a las mutaciones del ecosistema actual. "Los postulados de McLuhan y Fiori invocando la imprenta como un recurso repetitivo han devenido una estructura de yuxtaposiciones múltiples en la que la figura del creador se imbrica con la del receptor en el proceso de producción" (Cordón, Gómez y Alonso, 2011, p. 231). Aunque a ritmos distintos, la industria española de la prensa y del libro acometen su reto más importante.

\section{Referencias}

Aguado Terrón, J. M., Feijoo González, C. y Martínez Martínez, I. J. (2013). La comunicación móvil: hacia un nuevo ecosistema digital. Barcelona: Gedisa. 
Aguado, J. M. y Martínez, I. (2010). Construyendo la cuarta pantalla. Percepciones de los actores productivos del sector de las comunicaciones móviles. Telos, 83.

AEDE (2007). Libro Blanco de la Prensa Diaria. Madrid: AEDE.

AEDE (2014). Libro Blanco de la Prensa Diaria. Madrid: AEDE.

Ahonen, T. (2008). Mobile as 7th of the mass media: cellphone, cameraphone, iPhone, Smartphone. Londres: Futuretext.

Barandiarán, J. M., Gil, M. y Ortuño, M. (2014). El libro: crisis analógica y emergencia digital. En E. Bustamante y F. Rueda, Informe sobre el estado de la cultura en España: la salida digital (pp. 53-64). Madrid: Fundación Alternativas.

Boix Domenech, R. y Lazzeretti, L. (2012). Las industrias creativas en España: una panorámica. Investigaciones Regionales, 22, 181-206.

Burk, R. (2001). E-book devices and the marketplace: in search of customers. Library hi Tech, 19(4), 325-331.

Capos Freire, F. (2010). Los nuevos modelos de gestión de las empresas mediáticas. Estudios sobre el Mensaje Periodístico, 16, 13-30.

Canavilhas, J. (2009). Contenidos informativos para móviles: estudio de aplicaciones para iPhone. Revista Textual \& Visual Media, 2, 6180. Recuperado el 20 de enero de 2015 de http://ubithesis.ubi. pt/bitstream/10400.6/684/1/canavilhas-j-contenidos-informativos-para-moviles.pdf

Canavilhas, J. (2012). Jornalismo para dispositivos móveis: informação hipermultimediática e personalizada. En Actas do IV CICLCS. Recuperado el 20 de enero de 2015 de http://bocc.ubi.pt/pag/ canavilhas-joao-jornalismo-para-dispositivos-moveis.pdf 
Casero-Ripollés, A. (2010). Prensa en internet: nuevos modelos de negocio en el escenario de la convergencia. El Profesional de la Información, 19(6), 595-601. Recuperado el 25 de enero de 2015 de http://core.ac.uk/download/pdf/11887197.pdf

Castells, M., Fernández-Ardèvol, M., Linchuan Qiu, J. y Sey, A. (2007). Comunicación móvil y sociedad: una perspectiva global. Barcelona: Ariel-Fundación Telefónica.

Castells, M., Caraça, J. y Cardoso, M. (2013). Después de la crisis. Madrid: Alianza.

Celaya, J., Rausell, P. y Villaroya, A. (2013). La internacionalización de las industrias culturales y creativas españolas. Madrid: Fundación Alternativas.

Codina, L. (1998). El libro digital y el futuro de la edición. El Profesional de la Información, 7(1-2). Recuperado el 25 de enero de 2015 de http: / / www.elprofesionaldelainformacion.com/contenidos/1998/enero/el_libro_digital_y_el_futuro_de_la_edicion.html

Cordón, J. A., Gómez, R. y Alonso, J. (2011). Gutenberg 2.0. La revolución de los libros electrónicos. Gijón: Trea.

Cordón, J. A., Alonso, J. y Martín, H. (2010). Los libros electrónicos: la tercera ola de la revolución digital. Anales de Documentación, 13, 53-80.

Corredor, P. (2013). El estado de la cultura en España. Madrid: Fundación Alternativas.

Costa-Knufinke, J. (2010). Adaptación de las editoriales españolas al libro electrónico. El Profesional de la Información, 19(1), 13-20.

Costa-Sánchez, C. (2012). Ciberperiodismo en el smartphone: estudio de la multimedialidad, usabilidad, hipertextualidad e interactividad 
de las aplicaciones de medios nativos digitales para smartphone. Estudios sobre el Mensaje Periodístico, 18, 243-251.

Costa-Sánchez, C. (2013). Prensa en el smartphone: modelos aplicados en los diarios españoles y necesidades de desarrollo. Icono 14, 11 (2), 2-24.

Costa-Sánchez, C. (2014). Las singularidades del medio móvil: integración multimedia, personalización, geolocalización y participación. Estudio de su presencia en las apps de la prensa española. Palabra Clave, 17(3), 672-694.

Costa-Sánchez, C., Rodríguez-Vázquez, A. I. y López-García, X. (2015). Del periodismo transmedia al replicante: cobertura informativa del contagio de ébola en España por elpais.com. El Profesional de la Información, 24(3), 1699-2407.

Curran,J. (2010). The future of journalism. Journalism Studies, 11(4), 464-476.

Díaz-Noci, J. (2010). Medios de comunicación en internet: algunas tendencias. El Profesional de la Información, 19(6), 561-567.

Díaz Nosty, B. (2011). Libro negro del periodismo en España. Madrid: Asociación de la Prensa de Madrid/Cátedra Unesco de Comunicación de la Universidad de Málaga.

Díaz Nosty, B. (2013). La prensa en el nuevo ecosistema informativo. ; Que paren las rotativas! Madrid: Fundación Telefónica.

Federación de Gremios de Editores de España (2008). Barómetro de hábitos de lectura y compra de libros tercer trimestre de 2008. Recuperado el 12 de febrero de 2015 de http://www.federacioneditores.org/0 Resources/Documentos/NP_Lectura_2008_v2.pdf

Federación de Gremio de Editores de España (2012). Barómetro de hábitos de lectura y compra de libros. Recuperado el 12 de febrero de 2015 de http://cdn.20m.es/adj/2013/02/07/2076.pdf 
Federación de Gremio de Editores de España (2013). Comercio interior del libro en España 2013. Madrid: Federación de Gremios de Editores de España.

Gómez-Escalonilla, G. (2005). El libro y la industria cultural. Madrid: Fundación Alternativas.

Gómez-Escalonilla, G. (2012). La industria del libro ante el reto digital.En F. Salgado y V. Martínez, Economía de la cultura y la comunicación en la era digital (pp. 541-563). Lisboa: Media XXI.

Guallar,J. (2013). Prensa digital en 2011-2012. Anuario ThinkEPI, 7, 194-199.

Instituto Nacional de Estadística (INE) (2014). Encuesta sobre equipamiento y uso de tecnologías de información y comunicación en los hogares. Recuperado el 18 de febrero de 2015 de http: / / www. ine.es/prensa/np864.pdf

Islas-Carmona, O. (2008). El prosumidor: el actor comunicativo de la sociedad de la ubicuidad. Palabra Clave, 11(1), 29-39. Recuperado el 18 de febrero de 2015 de http://www.scielo.org.co/pdf/pacla/ v11n1/v11n01a03.pdf

Jung, J., Chan-Olmsted, S., Park, B. y Kim, Y. (2012). Factors affecting ebook reader awareness, interest, and intention to use. New Media \& Society, 14(2), 204-224.

Marqués Pascual, J. (2012). La caída de difusión en los diarios de pago: principales causas y su relevancia en el caso español (Tesis de doctorado, Universitat Ramón Llull).

Maynard, S. (2010). The impact of e-books on young children's reading habits. Publishing Research Quarterly, 26(4), 236-248.

Meso Ayerdi, K., Larrondo Ureta, A., Peña Fernández, S. y Rivero Santamarina, D. (2014). Active audiences in the mobile ecosystem: Analysis 
of the interaction options in Spanish cybermedia through websites, mobile telephones and tablets. Hipertext. net, 12.

Meyer, P. (2006). The vanishing newspaper: Saving journalism in the information age. Columbia: University of Missouri Press.

Observatorio de la Lectura y el Libro (2014). Panorámica de la edición española de libros 2013. Recuperado el 5 de marzo de 2015 de http:// www.mecd.gob.es/cultura-mecd/areas-cultura/libro/mc/pee/ contenedora/presentacion.html

Observatorio de la Lectura y el Libro (2015). El sector del libro en España (2013-2015). Recuperado el 5 de marzo de 2015 de http://www. mecd.gob.es/cultura-mecd/dms/mecd/cultura-mecd/areas-cultura/libro/mc/observatoriolect/redirige/estudios-e-informes/ elaborados-por-el-observatoriolect/sector-libro-abril2015.pdf

Picard, R. (2010). Value creation and the future of news organizations: Why and how journalism must change to remain relevant in the twenty-first century. Lisboa: Media XXI.

Rodríguez, D. y Valderriolla, J. (2013). Metodología de la Investigación. Barcelona: UOC. Recuperado el 7 de marzo de 2015 de http:/ / zanadoria.com/syllabi/m1019/mat_cast-nodef/PID_00148556-1.pdf

Salaverría, R. (2010). Estructura de la convergencia. En X. López, X. Pereira, Convergencia digital: reconfiguración de los medios de comunicación en España (pp. 27-40). Santiago de Compostela: Universidad de Santiago de Compostela.

Sánchez, E. y Aguado, J. M (2010, 3-5 febrero). Dispositivos móviles y convergencia digital en los grupos de comunicación españoles: la perspectiva de los profesionales. En II Congreso Internacional AE-IC: Comunicación y desarrollo en la era digital. Málaga. 
Sanjuán, A., Nozal, T. y González, A. (2013). Usabilidad, interactividad y contenidos multimedia en la prensa para iPad: el caso de El País, El Mundo, ABC y La Razón. En Canavilhas, J. (coord.), Notícias e Mobilidade: O Jornalismo na Era dos Dispositivos Móveis (pp. 7398). Covilha: Labcom.

Scolari, C. A., Navarro Güere, H., Pardo, H., García Medina, I. y Soriano Clemente, J. (2009). Comunicación móvil: actores y producción de contenidos en Cataluña. Comunicación y Sociedad, 22(2), 159-185.

Scolari, C. A. (2008). Hipermediaciones: elementos para una teoría de la comunicación digital interactiva. Barcelona: Gedisa.

Siegenthaler, E., Wurtz, P. y Groner, R. (2010). Improving the usability of e-book readers. Journal of Usability Studies, 6(1), 25-38.

Silva Rodríguez, A. (2013). Os cibermedios nos dispositivos móviles (Tesis de doctorado, Universidad de Santiago de Compostela, Santiago de Compostela).

Vacas, F. (2007). Teléfonos móviles: la nueva ventana para la comunicación integral. Madrid: Creaciones Copyright.

VV. AA. (2015). Sociedad de la Información en España 2014. Fundación Telefónica Recuperado el 7 de marzo de 2015 de http://www. fundaciontelefonica.com/arte_cultura/publicaciones-listado/ pagina-item-publicaciones/?itempubli=323

WAN-IFRA (2015). Trends in newsrooms 2015. Frankfurt: Wan/Ifra.

Westlund, O. (2013). Mobile news. Digital Journalism, 1(1), 6-26. 